To appear in The Astronomical Journal

Preprint typeset using $\mathrm{LT}_{\mathrm{E}} \mathrm{X}$ style emulateapj v. 5/2/11

\title{
STAR CLUSTERS IN M 31. V. EVIDENCE FOR SELF-ENRICHMENT IN OLD M31 CLUSTERS FROM INTEGRATED SPECTROSCOPY
}

\author{
RicARdo P. SCHIAVON \\ Astrophysics Research Institute, Liverpool John Moores University, 146 Brownlow Hill, Liverpool, L3 5RF, United Kingdom.
}

Nelson Caldwell

Harvard-Smithsonian Center for Astrophysics, 60 Garden Street, Cambridge, MA 02138, USA

Charlie Conroy

Department of Astronomy \& Astrophysics, University of California, Santa Cruz, CA, USA

Genevieve J. Graves

Department of Astrophysical Sciences, Princeton University, Princeton, NJ, USA

JAY STRADER

Department of Physics and Astronomy, Michigan State University, East Lansing, MI 48824, USA

Lauren A. MacArthur

National Research Council of Canada, Victoria, BC V9E 2E7, Canada

Stéphane Courteau

Department of Physics, Engineering Physics \& Astronomy, Queens University, Kingston, Ontario, Canada

AND

PAUl HaRding

Department of Astronomy, Case Western Reserve University, Cleveland, OH 44106-7215, USA

To appear in The Astronomical Journal

\begin{abstract}
In the past decade, the notion that globular clusters (GCs) are composed of coeval stars with homogeneous initial chemical compositions has been challenged by growing evidence that they host an intricate stellar population mix, likely indicative of a complex history of star formation and chemical enrichment. Several models have been proposed to explain the existence of multiple stellar populations in GCs, but no single model provides a fully satisfactory match to existing data. Correlations between chemistry and global parameters such as cluster mass or luminosity are fundamental clues to the physics of GC formation. In this Letter, we present an analysis of the mean abundances of $\mathrm{Fe}, \mathrm{Mg}$, $\mathrm{C}, \mathrm{N}$, and $\mathrm{Ca}$ for 72 old GCs from the Andromeda galaxy. We show for the first time that there is a correlation between the masses of GCs and the mean stellar abundances of nitrogen, spanning almost two decades in mass. This result sheds new light on the formation of GCs, providing important constraints on their internal chemical evolution and mass loss history.
\end{abstract}

Subject headings: globular clusters: general

\section{INTRODUCTION}

Globular clusters (GCs) are the oldest known stellar systems and their study has contributed fundamental knowledge to our understanding of stellar evolution and galaxy formation (e.g., Searle \& Zinn 1978; Ashman \& Zepf 1992; Brodie \& Strader 2006). Yet their origin remains poorly understood. The discovery of multiple stellar populations with a range of chemical compositions in Milky Way (MW) GCs revolutionized our understanding of GC formation, as these systems were previously believed to form in a single rapid star formation event. Several scenarios were proposed

R.P.Schiavon@ljmu.ac.uk to explain the observed complexities of the stellar populations in GCs (e.g., Renzini 2008; D'Ercole et al. 2010; Decressin et al. 2007; De Mink et al. 2009; Marcolini et al. 2009; Valcarce \& Catelan 2011; Conroy \& Spergel 2011), but all fail to match all the observations of Galactic GCs. In common to most models is the following triad of features: 1) GCs underwent internal chemical evolution; 2) more massive GCs self-enriched more efficiently due to an increased ability to hold on to enriched gas for subsequent star formation; and 3) GCs have lost most of their primordial mass.

It is difficult to use resolved observations of Galactic GCs to submit the above predictions to quantitative scrutiny, as this requires the determination of detailed 
abundance patterns for statistically significant samples of individual GC stars spanning a wide range of $\mathrm{GC}$ masses. Such determinations are very costly, as they rely on high-resolution spectroscopy based on 8-10 m telescopes. Integrated spectroscopy can provide important supplementary information to constrain models, in the form of mean abundances of elements such as nitrogen, which indicate the presence of self-enrichment in GCs. Based on a sample of 72 old GCs belonging to the Andromeda (M31) galaxy we study the correlations between $\mathrm{GC}$ mass and the mean abundances of $\mathrm{Fe}, \mathrm{Mg}, \mathrm{C}, \mathrm{N}$, and $\mathrm{Ca}$. For the first time, we find evidence for the presence of a correlation between GC mass and the mean abundance of nitrogen. This result challenges the notion that GCs have lost most of their masses since formation, and may help constrain the source of chemical enrichment in these systems.

This Letter is organized as follows: the data are described in Section 2 the results are presented in Section 3. and our conclusions are summarized in Section 4 .

\section{DATA}

The M31 GC spectra employed in this analysis Caldwell et al. 2009) were obtained with the MMT/Hectospec spectrograph, at Mount Hopkins, Arizona, whereas the spectra of MW GCs were collected with the Boller \& Chivens spectrograph attached to the Blanco $4 \mathrm{~m}$ telescope at Cerro Tololo Interamerican Observatory (Schiavon et al. 2005). Absorption line indices, measured in the Lick system, as well as internal and systematic uncertainties, are discussed in detail elsewhere (Schiavon et al. 2012).

\subsection{Elemental Abundances}

Lick index measurements are converted into abundance and age estimates, along with associated uncertainties, using the EZ_Ages code (Graves \& Schiavon 2008), which implements a method developed by Schiavon (2007). For more details on abundance error estimates, including extensive Monte Carlo simulations, see Graves \& Schiavon (2008). Nitrogen abundances are based on the $\mathrm{CN}_{1}$ and/or $\mathrm{CN}_{2}$ indices, and because the $\mathrm{CN}$ band measured by these indices is too weak to be detected in our medium resolution integrated spectra of GCs with $[\mathrm{Fe} / \mathrm{H}] \lesssim-1.0$ (Schiavon et al. 2012), only GCs more metal-rich than that limit are considered. Thus, our initial sample includes $175 \mathrm{GCs}$ with iron abundance within the range $-1.0 \leq[\mathrm{Fe} / \mathrm{H}] \leq+0.2$. The sample is further restricted to $\overline{G C s}$ for which our spectra have $\mathrm{S} / \mathrm{N} \geq 100 / \AA$, for which our abundances, particularly for nitrogen, are most reliable. The final sub-sample of 72 GCs spans masses between $10^{4.8}$ and $10^{6.5} M_{\odot}$. Caldwell et al. (2009) and Schiavon et al. (2012) showed that M31 GCs are about as old as their MW couterparts, thus the two GC systems can be compared on the same footing.

\subsection{Cluster Masses}

Mass is a key parameter in our analysis, and we note that elemental abundances and masses are based on independent data and methods. Within the sample of 72 GCs studied in this Letter, dynamical masses $\left(M_{d y n}\right)$ from Strader et al. (2011) are available for

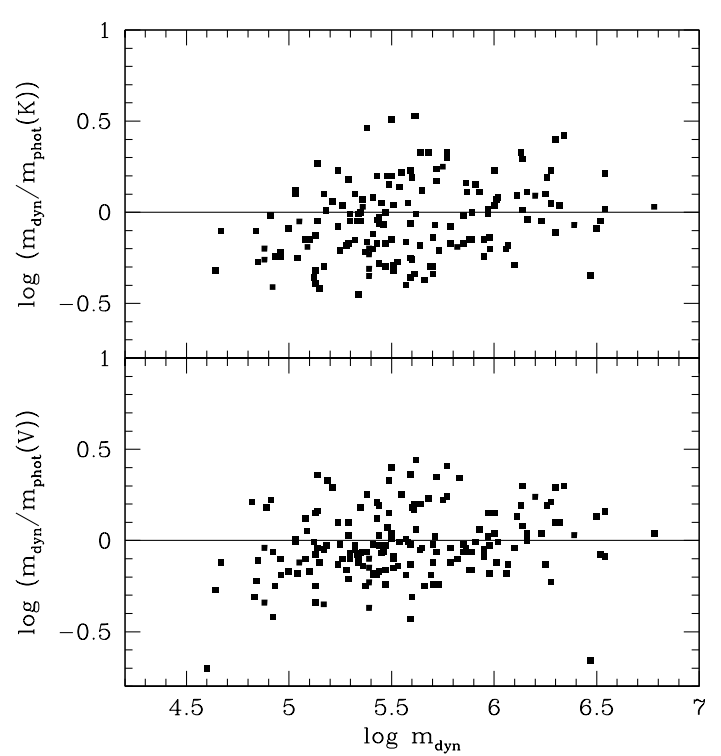

FIG. 1.- Comparison between dynamical and photometric masses, based on K- (top panel) or V-band (bottom panel) magnitudes. The three sets of GC masses are in very good agreement, without significant zero point differences.

53 objects and, for the remaining GCs, photometric masses $\left(M_{\text {phot }}\right)$ are adopted, estimated assuming $m-$ $M=24.43$ and $\mathrm{M} / \mathrm{L}_{V}=1.5$ or $\mathrm{M} / \mathrm{L}_{K}=0.8$, depending on available photometry. Masses for MW GCs were taken from McLaughlin \& van der Marel (2005) and Gnedin \& Ostriker (1997).

We next check for the presence of systematic effects on $M_{\text {phot }}$ due to the impact of age and/or chemical composition on mass-to-light ratios. In Figures 1 and 2 , $\mathrm{V}$ bandand $\mathrm{K}$ band-based $M_{\text {phot }} \mathrm{s}$ are compared with $M_{d y n}$ as a function of $M_{d y n}$ and [Fe/H], for a larger GC sample from Caldwell et al. (2009). Both sets of photometric masses are in good agreement with $M_{d y n}$ and in Figure 1 the residuals show at most a very slight correlation with GC mass, which is negligible for the purposes of this Letter. In Figure 2 a trend can be seen with [Fe/H], particularly in the case of $\mathrm{K}$ band-based photometric masses. The effect is very small, however, especially considering the $[\mathrm{Fe} / \mathrm{H}]$ range of the sample $(-1.0 \leq[\mathrm{Fe} / \mathrm{H}] \leq+0.2)$, and the fact that photometric masses were adopted for only $\sim 1 / 4$ of those GCs. Therefore, systematics on the $\mathrm{K}$ band-based photometric masses is at most of the order of 0.2 dex, and half of that in the case of $\mathrm{V}$ band-based masses. Considering the dynamic range (in mass) of our correlations, this effect is negligible. In fact, the statistics on the correlations studied in this Letter is unaffected regardless of whether we employ only V-band or K-band photometric masses. We conclude that our analysis incurs no important systematic effects due to the adoption of photometric masses for part of the sample. For an in depth discussion of Figures 1 and 2, see Strader et al. (2011).

\section{RESULTS}




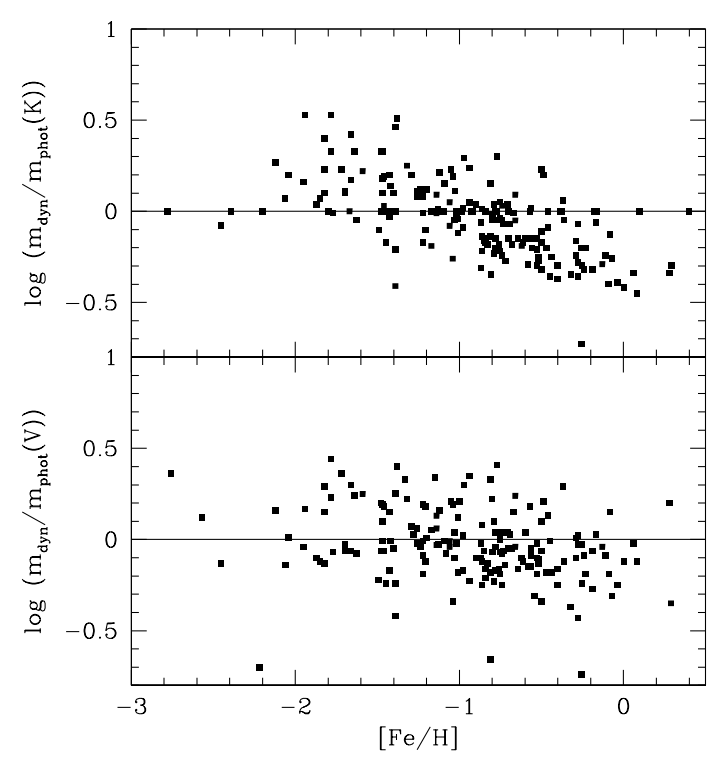

FIG. 2.- Comparison between dynamical and photometric masses, as a function of $[\mathrm{Fe} / \mathrm{H}]$. Photometric masses are slightly smaller at higher $[\mathrm{Fe} / \mathrm{H}]$, but the effect is negligible.

Resulting $[\mathrm{Fe} / \mathrm{H}]$ and abundance ratios $([\mathrm{X} / \mathrm{Fe}])$ are plotted as a function of GC mass in Figure 3, where GCs are color coded according to $[\mathrm{Fe} / \mathrm{H}]$. Squares (open stars) represent data for M31 (MW) GCs. The Spearman rank correlation coefficient $(\rho)$ and the probability associated with the null hypothesis $(\mathrm{P})$ for a correlation between data pairs are shown in the upper left corner of each panel. The only quantity correlating significantly with GC mass is $[\mathrm{N} / \mathrm{Fe}]\left(\rho=0.37, P=2 \times 10^{-3}\right)$. We note that $[\mathrm{N} / \mathrm{Fe}]$ correlates even more strongly with GC absolute magnitude, $\mathrm{M}_{V}$, which is a more directly measurable quantity, more reliable than mass $(\rho=-0.47$, $P=1 \times 10^{-4}$, not shown). The numbers suggest the presence of a weak anti-correlation between $[\mathrm{C} / \mathrm{Fe}]$ and mass, although that is not confirmed by visual inspection of the data.

Most of the scatter in the $[\mathrm{N} / \mathrm{Fe}]-$ mass relation is due to a (stronger) correlation between $[\mathrm{N} / \mathrm{Fe}]$ and $[\mathrm{Fe} / \mathrm{H}]$, which can be perceived in Figure 3, where higher $[\mathrm{Fe} / \mathrm{H}]$ GCs have systematically higher $[\mathrm{N} / \mathrm{Fe}]$ at fixed mass. A similar, although steeper, correlation was found in earlytype galaxies (Schiavon 2007), which was confirmed independently by Conroy et al. (2013). No such correlation can be seen in solar neighborhood field star data compiled by Schiavon (2007), although this may be due to inhomogeneous abundance data. Because GCs in the high $[\mathrm{Fe} / \mathrm{H}]$ bin span a limited mass range, they only contribute vertical scatter to the $[\mathrm{N} / \mathrm{Fe}]$-mass relation at $5.5 \lesssim \log M / M_{\odot} \lesssim 6.0$, leading to a spurious degradation of the correlation. Therefore, an accurate assessment of the $[\mathrm{N} / \mathrm{Fe}]$-mass correlation requires restricting the sample to GCs with $-1.0<[\mathrm{Fe} / \mathrm{H}]<-0.4(61 \mathrm{GCs})$, as shown in the upper left panel of Figure 4. The statistical significance of the $[\mathrm{N} / \mathrm{Fe}]-\operatorname{mass}\left([\mathrm{N} / \mathrm{Fe}]-\mathrm{M}_{V}\right)$ increases in this case, with $\rho=0.49(\rho=-0.51)$. Figure 4 also shows linear fits to the relations of $[\mathrm{N} / \mathrm{Fe}]$ with mass and
$[\mathrm{Fe} / \mathrm{H}]$ for M31 GCs (left panels). The residuals from the $[\mathrm{N} / \mathrm{Fe}]-$ mass relation correlate with $[\mathrm{Fe} / \mathrm{H}]$ (upper right), whereas those from the $[\mathrm{N} / \mathrm{Fe}]-[\mathrm{Fe} / \mathrm{H}]$ relation correlate with mass (lower right), providing additional evidence to the presence of a $[\mathrm{N} / \mathrm{Fe}]$-mass relation.

For a small sample of Galactic GCs, abundance studies of main sequence stars show a large spread in $[\mathrm{N} / \mathrm{Fe}]$ and $[\mathrm{C} / \mathrm{Fe}]$ (e.g., Briley et al. 2004). Because the atmospheres of dwarf stars are not affected by internal mixing from evolutionary effects, this spread is usually interpreted as resulting from chemical evolution within the GCs' stellar populations, with the first generation stars having an abundance pattern similar to that of field populations of same $[\mathrm{Fe} / \mathrm{H}]$, and stars formed subsequently being enhanced in nitrogen and depleted in carbon. While observational challenges prevent abundance measurements in individual M31 GC stars, they are likely to be present there as well, thus our abundance ratio measurements should be interpreted as mean values. Evidence from MW GCs suggests that second generation stars represent a significant, approximately constant, fraction of GC masses today (e.g., Carretta et al. 2010a; Valcarce \& Catelan 2011; Conrov \& Spergel 2011). Therefore mean abundances based on integrated light spectroscopy provide a reliable indication of the amount of chemical enrichment undergone by GCs. Thus, the correlation between GC mass and mean $[\mathrm{N} / \mathrm{Fe}]$ is evidence for the occurence of a feedback-regulated history of star formation and chemical enrichment in GCs. A somewhat loose correlation between $\mathrm{GC}$ absolute magnitude and the range of $[\mathrm{O} / \mathrm{Na}]$ in GC stars in a smaller sample of MW GCs was found before (Carretta et al. 2010a). However, the correlation presented in this Letter is more robust, based on a larger sample, and for the first time for nitrogen, which is difficult to measure in large samples of individual stars.

Average formal uncertainties in abundances (lower right corner of each panel of Figure 3) are small, but zero point uncertainties may be larger. Systematic effects may affect $[\mathrm{N} / \mathrm{Fe}]$ values, especially for $[\mathrm{N} / \mathrm{Fe}] \gg$ +0.3 , where they rely on extrapolations of the $\mathrm{CN}_{1}$ index sensitivity calculations (Schiavon 2007). Further uncertainties stem from $[\mathrm{N} / \mathrm{Fe}]$ being derived from the strengths of $\mathrm{CN}$ bands, which are also sensitive to $[\mathrm{C} / \mathrm{Fe}]$, although the latter are well constrained by modeling of the $\mathrm{C}_{2} 4668$ index. Most importantly, although model uncertainties may render the absolute values of $[\mathrm{N} / \mathrm{Fe}]$ in Figure 3 somewhat uncertain, the correlation between $[\mathrm{N} / \mathrm{Fe}]$ and $\mathrm{GC}$ mass seems robust. A substantial expansion of the data on $[\mathrm{C} / \mathrm{Fe}]$ and $[\mathrm{N} / \mathrm{Fe}]$ towards large samples of individual MW GC stars would help validate the existence of this trend and uncover its origin.

Additional, model independent, evidence comes from correlations between line indices and GC mass. The correlations are shown in Figure 5, where selected line indices are plotted against GC mass, for the same sample from Figures 3 and 4 . We find statistically significant correlations between GC mass and $\mathrm{CN}$ indices, including Lick $\mathrm{CN}_{1}$ (and $\mathrm{CN}_{2}$, not shown) and CN3883 (Davidge \& Clark 1994). The CN bands are sensitive to the abundances of nitrogen and carbon, but since there is no correlation between $\mathrm{C}_{2} 4668$ and GC mass, one concludes that the correlations are driven by nitrogen. A statistically significant correlation is also found between 

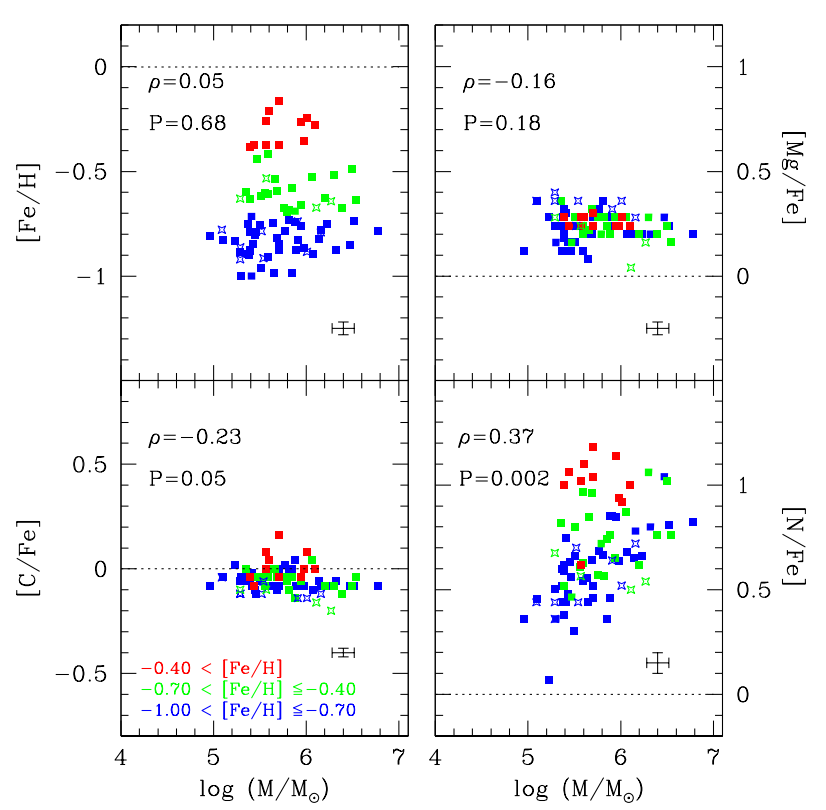

FIG. 3.- Globular clusters are displayed in several masschemistry planes. Mean iron abundance and abundance ratios are plotted for M31 (gray squares) and MW GCs (stars). Vertical scales span the same dynamic range in all panels, for an immediate visual assessment of the relative strength of correlations and amount of scatter in the data. Average error bars are displayed on the lower right corner of each panel. Dotted lines indicate the solar value. The Spearman rank correlation coefficient for each pair, and the probability associated with the null hypothesis are displayed on the top left corner of each panel. Our main result is the correlation between $[\mathrm{N} / \mathrm{Fe}]$ and $\mathrm{GC}$ mass (bottom right panel), which is consistent with the presence of self-enrichment in M31 GCs. The correlation is more obvious at fixed $[\mathrm{Fe} / \mathrm{H}]$, due to a concurrent correlation between $[\mathrm{N} / \mathrm{Fe}]$ and $[\mathrm{Fe} / \mathrm{H}]$ (see Figure 4 ). The absence of a correlation in heavy elements such as $\mathrm{Fe}, \mathrm{Mg}$, and $\mathrm{Ca}$ indicates that $\mathrm{N}$ enrichment cannot be caused by explosive nucleosynthesis. The numbers suggest the presence of a weak anti-correlation between $[\mathrm{C} / \mathrm{Fe}]$ and mass, which is not confirmed by visual inspection of the data.

GC mass and the $\mathrm{NaD}$ index, which is sensitive to sodium abundance - the elemental abundance whose variations constitute most of the evidence for self-enrichment in Galactic GCs (e.g., Carretta et al. 2010a). The absence of correlations between mass and indices such as $\langle F e\rangle$ and $\mathrm{Mg} b$ (not shown), which are mostly sensitive to the abundances of iron and magnesium, respectively, further corroborates our conclusion that only the abundances of specific elements, such as nitrogen, and possibly sodium, correlate with GC mass.

The correlations between GC mass and line indices are unsurprisingly noisier than that between $[\mathrm{N} / \mathrm{Fe}]$ and GC mass, since line indices are not uniquely determined by the abundance of the target elements, being also affected by other parameters, such as age, metallicity, HB morphology, and other elemental abundances. The strength of the correlation is weakest for CN3883 index, for unclear reasons, which may be associated with lower $\mathrm{S} / \mathrm{N}$ blueward of $4000 \AA$ and possibly the influence of a spread in horizontal branch morphology at fixed chemical composition and age. The latter effect should impact more strongly the violet CN3883 index than its counterpart at $4170 \AA$.
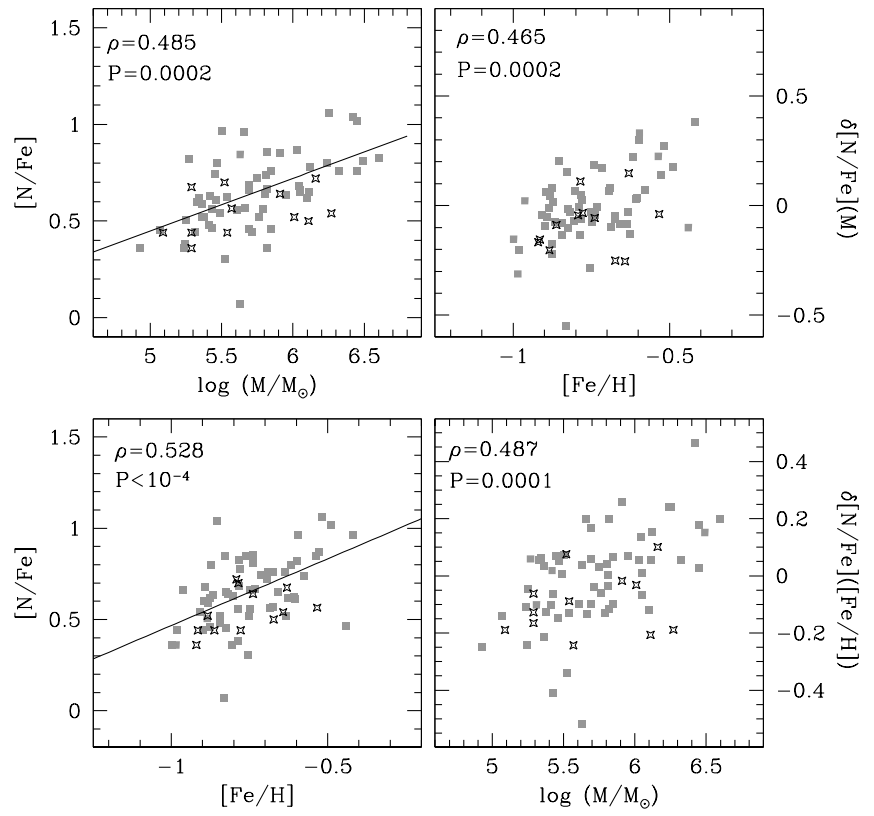

FIG. 4.- The left panels show linear fits to the relations between $[\mathrm{N} / \mathrm{Fe}]$ and GC mass (top, repeated from Figure 3) and [Fe/H] (bottom). The right panels plot the residuals from each of these fits as a function of mass (bottom) and $[\mathrm{Fe} / \mathrm{H}]$. Symbols as in Figure 3 The residuals from the fit to the $[\mathrm{N} / \mathrm{Fe}]$-mass relation correlate with $[\mathrm{Fe} / \mathrm{H}]$. Conversely, the residuals from the $[\mathrm{N} / \mathrm{Fe}]-[\mathrm{Fe} / \mathrm{H}] \mathrm{re}-$ lation correlate with mass. The correlation between mass and the residuals from the $[\mathrm{N} / \mathrm{Fe}]-[\mathrm{Fe} / \mathrm{H}]$ relation provides further evidence for the presence of self-enrichment in GCs. On the other hand, the correlation between the residuals from the $[\mathrm{N} / \mathrm{Fe}]$-mass relation and $[\mathrm{Fe} / \mathrm{H}]$ accounts for most of the large scatter in that relation. The correlation between $[\mathrm{N} / \mathrm{Fe}]$ and $[\mathrm{Fe} / \mathrm{H}]$ argues for the presence of secondary N-enrichment, which may help constrain the source of chemical enrichment in GCs.

\section{DISCUSSION}

Correlations between cluster mass and mean abundances of $\mathrm{N}$ and (possibly) $\mathrm{Na}$ have the following implications for our understanding of the formation of GCs and the MW halo. First, they are consistent with the occurrence of a feedback-regulated history of star formation and chemical enrichment in M31 GCs. One would thus expect that the correlation breaks down at some low mass limit, below which the gravitational potential would be too shallow to retain chemically enriched ejecta. No such effect is detected, which may be due to the relatively high low-mass limit of our high $\mathrm{S} / \mathrm{N}$ sample, $\mathrm{M}>10^{4.8} \mathrm{M}_{\odot}$, which is higher than the low mass threshold for GC self-enrichment in M31, $\sim 10^{4.5} \mathrm{M}_{\odot}$, proposed by Conroy \& Spergel (2011) on the basis of modeling of pressura balance in intracluster gas in the early galaxy environment. Better data are required for lower mass old GCs, to test that prediction.

Second, many authors (e.g., Renzini 2008; Valcarce \& Catelan 2011; Conroy 2012) proposed that MW GCs were as much as 10-100 times more massive in the past, with most of their stellar masses being lost through two-body relaxation and tidal stripping during interactions with the Galactic gravitational potential. The discovery of CN-strong stars in the MW halo field led to the suggestion that a substantial fraction of the stellar halo results from GC dissolution 
(Martell et al. 2011; Carollo et al. 2013). However, one intuitively expects that copious mass loss erase pre-existing correlations between chemistry and mass, unless, on the one hand, most mass loss happened before the bulk of internal chemical evolution took place in GCs or, on the other, mass loss physics establishes a smooth relation between primordial and current GC masses. On the one hand, numerical simulations by Baumgardt \& Makino (2003) show that most stellar mass loss takes place over timescales of $10^{9} \mathrm{yr}$, which implies higher stellar age spreads than allowed by observations of turnoff stars in both MW and LMC GCs (e.g., Rubele et al. 2013). On the other hand, simulations suggest that mass loss is proportional to a power of cluster mass (Lamers et al. 2010), so that mass loss should preserve a pre-existing mass-chemical composition relation, provided it depends more strongly on GC mass than on other variables such as orbital characteristics and environment. Yet the latter dependencies may be important enough to account for the observed scatter in the $[\mathrm{N} / \mathrm{Fe}]$-mass relation.

Third, our results help constrain the nature of stars responsible for chemical enrichment in GCs. Four mechanisms have been proposed: winds from intermediatemass Asymptotic Giant Branch (AGB) stars (e.g., Renzini 2008; D'Ercole et al. 2010), rapidly rotating massive stars (e.g., Decressin et al. 2007), mass-transfer binary massive stars (e.g., De Mink et al. 2009), and explosive nucleosynthesis (e.g., Marcolini et al. 2009; Valcarce \& Catelan 2011, only for the most massive GCs). The correlation between $[\mathrm{N} / \mathrm{Fe}]$ and mass, and the absence of correlations for by-products of explosive nucleosynthesis, such as $\mathrm{Mg}$ (and Ca, not shown) suggests that the enrichment of the intracluster medium was caused by low energy ejecta. To first order, the velocity of retained stellar ejecta should be comparable to typical GC escape velocities, which range between a few and $\sim 100 \mathrm{~km} \mathrm{~s}^{-1}$ (Gnedin et al. 2002). This is in relatively good agreement with typical wind velocities of AGB stars (Dupree et al. 1992; Loup et al. 1993) and interacting-binary winds (De Mink et al. 2009). Likewise, these escape velocities rule out winds from massive stars, with typical velocities of order $10^{3} \mathrm{~km}$ $\mathrm{s}^{-1}$ (Kudritzki \& Puls 2000), although physical mechanisms such as wind-wind interactions in binary systems (Palouš et al. 2009) and slow winds from fast-rotating massive stars (Decressin et al. 2007) were proposed to address this tension. While our results for $\mathrm{Fe}, \mathrm{Mg}$, and $\mathrm{Ca}$ rule out enrichment by explosive nucleosynthesis it is conceivable that it plays a role in the most massive GCs in M31, since the theoretical expectation is for supernovae ejecta to be retained by clusters with $\mathrm{M} \gtrsim 10^{7} \mathrm{M}_{\odot}$ (Baumgardt et al. 2008). Those clusters are the analogs of massive MW GCs, such as $\omega$ Cen and M 54, whose stellar populations present a range of [Fe/H] (Pancino et al. 2002; Carreta et al. 2010b). The existence of a correlation between $[\mathrm{N} / \mathrm{Fe}]$ and $[\mathrm{Fe} / \mathrm{H}]$ is interesting. Carretta et al. (2009) also suggest the existence of a correlation between the amount of cluster selfenrichment (in $\mathrm{Al}$ ) and a combination of $[\mathrm{Fe} / \mathrm{H}]$ and $\mathrm{M}_{V}$. There is no evidence for a correlation between $[\mathrm{N} / \mathrm{Fe}]$ and $[\mathrm{Fe} / \mathrm{H}]$ in Galactic field stars, suggesting that it is an im- print of GC chemical evolution. This may be a clue to the
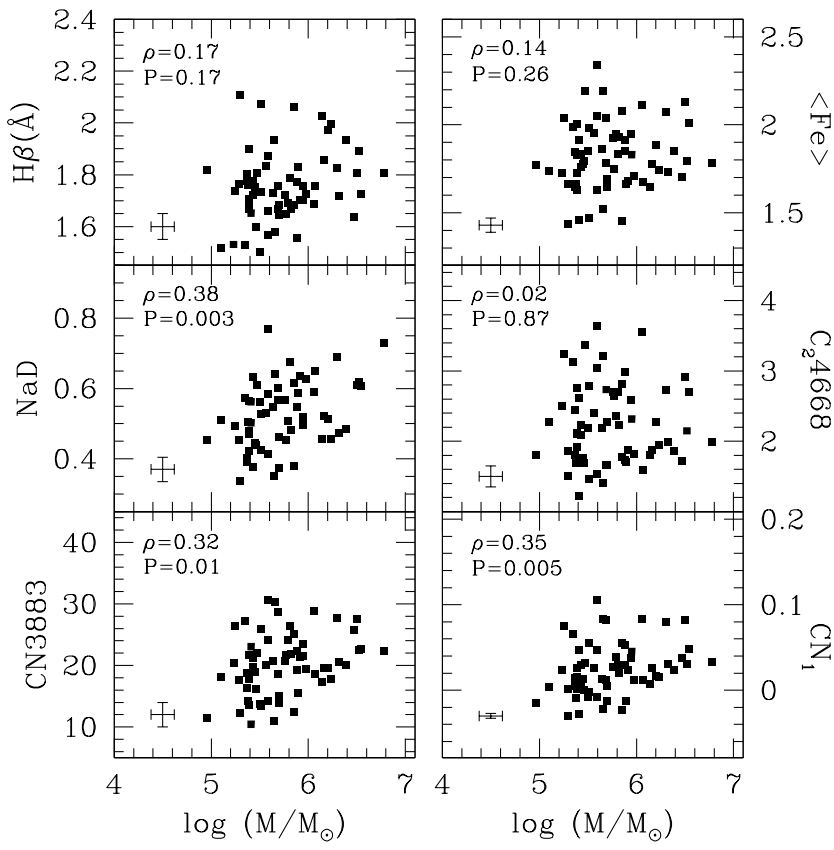

FIG. 5.- Correlations between line indices and GC mass. The sample is the same as used in Figures 3 and 4 Statistically significant correlations are found for indices sensitive to the abundances of nitrogen $\left(\mathrm{CN}_{1}\right.$ and $\left.\mathrm{CN} 3883\right)$ and sodium $(\mathrm{NaD})$, whereas indices mainly sensitive to age $(H \beta)$ or the abundances of iron $(\langle F e\rangle)$ and carbon $\left(C_{2} 4668\right)$ do not correlate with mass. Cluster mass correlate more weakly with line indices than with abundance ratios because the former are sensitive to other parameters, such as age, metallicity, and horizontal-branch morphology (especially in the case of CN3883). These plots corroborate our conclusion that GCs are self enriched in light elements, but not in by-produts of explosive nucleosynthesis.

source of chemical enrichment in these systems, possibly indicating the presence of secondary $\mathrm{N}$ enrichment.

Finally, from chemical evolution models based on enrichment from material processed through hot bottom burning in massive AGB stars (e.g., D'Ercole et al. 2010), one would expect the $[\mathrm{N} / \mathrm{Fe}]$-mass correlation to be accompanied by a $[\mathrm{C} / \mathrm{Fe}]$-mass anti-correlation. Statistics suggests the possible presence of a weak anticorrelation (Figure 3), but that is not confirmed by visual inspection of the data. However, the variation in mean $[\mathrm{C} / \mathrm{Fe}]$ may be small enough that it would require more accurate data for its detection.

The existence of a mass-chemical composition relation is a strong clue to the chemical evolution history of GCs, which can constrain GC formation theory. The data discussed here provide a proving ground to test quantitative model predictions, including a chemical evolution prescription based on state-of-the-art yields and a star formation history grounded on a realistic account of the physical conditions prevailing in primordial clusters.

The authors thank the anonymous referee for a timely and very helpful review. RPS thanks Bob Rood (in memoriam), Maurizio Salaris, Nate Bastian, and Inger Jørgensen for useful discussions. RPS thanks the support of Gemini Observatory, where part of this research was conducted. 


\section{REFERENCES}

Ashman, K. \& Zepf, S.E. 1992, ApJ, 384, 50

Baumgardt, H. \& Makino, J. 2003, MNRAS, 340, 227

Baumgardt, H., Kroupa, P. \& Parmentier, G. 2008, MNRAS, 384, 1231

Briley, M.M., Cohen, J.G. \& Stetson, P.B. 2004, AJ, 127, 1579

Brodie, J.P. \& Strader, J. 2006, ARA\&A44, 193

Caldwell, N., Harding, P., Morrison, H., Rose, J.A., Schiavon, R. \& Kriessler, J. 2009, AJ, 137, 94

Carollo, D., Martell, S.L., Beers, T.C. \& Freeman, K.C. 2013, ApJ, 769, 87

Carretta, E., Bragaglia, A., Gratton, R.G. \& Lucatello, S. 2009, A\&A, 505139

Carretta, E., Bragaglia, A., Gratton, R.G. Recio-Blanco, A., Lucatello, S., D'Orazi, V. \& Cassisi, S. 2010, A\&A, 516, A55

Carretta, E., Bragaglia, A., Gratton, R.G. et al.2010, A\&A, 520, 95

Conroy, C. 2012, ApJ, 758, 21

Conroy, C. \& Spergel, D.N. 2011, ApJ, 726, 36

Conroy, C., Graves, G. \& van Dokkum, P., 2013, ApJ, submitted, arXiv:1303.6629

D'Ercole, A., D'Antona, F., Ventura, P., Vesperini, E. \& McMillan, S.L.W. 2010, MNRAS, 407, 854

Davidge, T.J. \& Clark, C.C. 1994, AJ, 107, 946

De Mink, S.E., Pols, O.R., Langer, N. \& Izzard, R.G. 2009, A\&A, 507,1

Decressin, T., Meynet, G. Charbonnel, C., Prantzos, N. \& Ekström, S. 2007, A\&A1029, 1044

Dupree, A.K., Sasselov, D.D. \& Lester, J.B. 1992, ApJ 387, L85

Gnedin, O.Y. \& Ostriker, J.P. 1997, ApJ, 474, 233

Gnedin, O.Y., Zhao, H., Pringle, J.E., Fall, S.M., Livio, M. \& Meylan, G. 2002, ApJ, 568, L23
Graves, G.J. \& Schiavon, R.P. 2008, ApJS, 177, 446

Kudritzki, R.-P. \& Puls, J. 2000, ARA\&A, 38, 613

Lamers, H.J.G.L.M., Baumgardt, H. \& Gieles, M. 2010, MNRAS, 408,305

Loup, C., Forveille, T., Omont, A. \& Paul, J.F. 1993, A\&AS, 99, 291

Marcolini, A., Gibson, B.K., Karakas, A.I. \& Sánchez-Blázquez, P. 2009, MNRAS, 395, 719

Martell, S.L., Smolinski, J.P., Beers, T.C. \& Grebel, E.K. 2011, A\&A, 534, 136

McLaughlin, D.E. \& van der Marel, R.P. 2005, ApJS, 161, 304

Palouš, J., Wünsch, R., Tenorio-Tagle, T. \& Silich, S. 2009, in IAU Symp., 270, 267

Pancino, E., Pasquini, L., Hill, V., Ferraro, F.R. \& Bellazzini, M. 2002, ApJ, 586, L101

Renzini, A. 2008, MNRAS, 391, 354

Rubele, S., Girardi, L., Kozhurina-Platais, V., Kerber, L., Goudfrooij, P., Bressan, A. \& Marigo, P. 2013, MNRAS, 430, 2774

Schiavon, R.P. 2007, ApJS, 171, 146

Schiavon, R.P., Rose, J.A., Courteau, S. \& MacArthur, L.A. 2005, ApJS, 160, 163

Schiavon, R.P., Caldwell, N., Morrison, H., Harding, P., Courteau, S., MacArthur, L.A. \& Graves, G.J. 2012, AJ, 143, 14

Searle, L. \& Zinn, R. 1978, ApJ, 225, 357

Strader, J., Caldwell, N. \& Seth, A. 2011, AJ, 142, 8

Valcarce, A.A.R \& Catelan, M. 2011, A\&A, 533, 120 